\title{
UM ESTUDO SOBRE A INTRODUÇÃO DE UM ASSISTENTE VIRTUAL PARA SUPORTE À ESCRITA COLETIVA
}

\author{
Ms Alexandra Lorandi Macedo* \\ Alexandre Lorenzatti** \\ Dr Eliseo Berni Reategui ${ }^{* * * *}$ \\ $\mathrm{D}^{\text {ra }}$ Patricia Alejandra Behar ${ }^{* * * *}$
}

\begin{abstract}
Resumo: Este artigo apresenta um estudo sobre o desenvolvimento de um assistente virtual para o ETC - Editor de Texto Coletivo. A pesquisa é parte integrante dos estudos desenvolvidos pelo NUTED/UFRGS (Núcleo Tecnologia Digital aplicada à Educação/ Universidade Federal do Rio Grande do Sul). O agente tem por objetivo organizar as informações e coordenações de ações do estudante no ETC e apresentá-las ao professor, oferecendo a este, suporte para a gestão pedagógica e tendo como foco a interação e aprendizagem do aluno diante do processo coletivo.

Palavras-chave: Assistente Virtual; Editor de Texto Coletivo e Escrita Coletiva
\end{abstract}

\section{A STUDY ABOUT THE INTRODUCTION OF A VIRTUAL ASSISTENT TO SUPPORT COLLECTIVE WRITING}

\begin{abstract}
This paper presents a study on the development of a virtual assistant for the Collective Text Editor ETC. This research is part of the studies carried out at NUTED/UFRGS (Núcleo Tecnologia Digital aplicada à Educação/ Universidade Federal do Rio Grande do Sul). The agent has as a goal to organize information and coordinate the students' actions in the text editor, and to present this information to the teacher. Such an approach gives teachers the necessary support for pedagogical management, focusing on the students' interaction and learning in a collective process.
\end{abstract}

Keywords: Virtual Assistants, Collective Text Editor, Collective Writing

\section{Introdução}

O conceito de personificação diz respeito ao uso da figura humana em uma interface, normalmente na forma de um personagem cujo objetivo é monitorar as ações do usuário e guiá-lo na utilização do sistema. A utilização da figura humana para melhorar a comunicação com o usuário é baseada na premissa de que a interação com um assistente personificado torna a interface do sistema mais amigável e centraliza a atenção do usuário em torno deste personagem. A utilização da figura humana em interfaces é um tópico que ainda apresenta controvérsias (Kaasinen, 98). No entanto, alguns resultados

\footnotetext{
* Doutoranda em Informática na Educação (PGIE/UFRGS), Mestre em Educação (PPGEDU/UFRGS) e pesquisadora no NUTED (UFRGS). E-mail: alorandimacedo@gmail.com

** Aluno do Bacharelado em Ciência da Computação - Departamento de Informática - Universidade de Caxias do Sul (UCS). E-mail: alorenza@ucs.br

*** Doutor em Computação (Universidade de Londres), Professor Adjunto do Departamento de Informática - Universidade de Caxias do Sul (UCS). E-mail: ebreateg@ucs.br

***** Doutora em Ciência da Computação (UFRGS), professora da Faculdade e Pós-Graduação em Educação e do Pós-Graduação em Informática na Educação (UFRGS); Coordenadora do NUTED/UFRGS. E-mail: pbehar@terra.com.br
} 
científicos começam a ser apresentados indicando que a personificação, em determinadas condições, pode aumentar o envolvimento do usuário e facilitar sua interação com o sistema (Blom, 01). Resultados comerciais igualmente promissores também já são apontados. A assistente virtual Emily, por exemplo, permitiu que uma loja de flores online aumentasse suas vendas em até três vezes quando usuários consultavam a assistente (Wallace, 00). Várias empresas têm oferecido este tipo de recurso na estruturação de serviços de atendimento online automatizado, como é o caso da La Cantoche (www.cantoche.com), Oddcast (www.oddcast.com), entre outras.

Neste estudo, um assistente virtual foi projetado para dar suporte ao trabalho de professores que desenvolvem sua prática mediando a construção coletiva de textos. Este assistente é capaz de monitorar o trabalho dos alunos, organizando as informações e coordenando as ações (Piaget, 1973) desenvolvidas pelos estudantes dentro do Editor de Texto Coletivo. A apresentação destas informações aos professores favorece a gestão pedagógica, tendo como foco a interação do aluno diante do processo coletivo.

Este artigo apresenta o editor de texto coletivo ETC, para o qual o assistente virtual foi proposto. Em seguida, o projeto do assistente virtual é detalhando, apresentando as principais funções especificadas para o personagem. Por fim, o artigo apresenta conclusões e proposições para novos estudos.

\section{O Editor de Texto Coletivo}

A concepção e elaboração do editor ETC apoiaram-se na perspectiva de construção do conhecimento e coordenação de ações previstas na teoria piagetiana (Piaget, 1995; 1973). Com base neste princípio o desenvolvimento das ferramentas que compõem o Editor busca favorecer momentos de interação, confronto de idéias, compartilhamento de proposições e negociações acerca da temática abordada pelos usuários. A seguir, farse-á a apresentação das principais ferramentas do software.

Ao acessar o Editor de Texto Coletivo o usuário tem visão permanente da barra superior de ferramentas. Nela é possível acessar os Dados Pessoais, RoodaExata (editor de fórmulas matemáticas), Biblioteca, Fórum, Bate-papo, A2 (comunicador instantâneo para usuários on-line), Ajuda e Sair. Todos estes itens podem ser acessados independentemente da tela/hierarquia em que o usuário esteja (Figura 1). Assim que este acessa qualquer grupo do qual participa, aparecem todos os textos em que está cadastrado como participante, gerente de grupo ou texto. As opções "Criar Novo Texto" ou "Convidar Novos Usuários" são privilégios somente concedidos aos Gerentes de grupo ou texto. Ainda na tela inicial do Editor temos acesso ao Mural Geral. Este exibe informações trazidas pelo sistema a partir de alterações feitas em determinado texto, além de mensagens postadas pelos Gerentes do Texto ou Grupo. Os Lembretes estão disponíveis logo abaixo do Mural para que o usuário o utilize com anotações particulares. Outro recurso disponível é a busca de textos que facilita a localização de diferentes temas que estejam em produção no editor, independente do usuário estar participando do grupo ou não. Esta é uma oportunidade para o mesmo localizar e solicitar permissão para participar de assuntos de seu interesse.

$\mathrm{Na}$ Figura 1 encontra-se a tela de edição do texto. A barra superior é fixa, conforme descrito anteriormente. Logo abaixo está a régua de hierarquia de navegação. Conforme o usuário acessa seu grupo, texto..., a régua marca de maneira hierárquica tais escolhas e, a partir de um clique sobre cada uma das opções, é possível retornar à navegação bem como identificar facilmente a área do software em que o usuário se encontra no momento. O mural disponível da tela de Edição do Texto traz informações postadas pelo Gerente do Texto. Logo abaixo do Mural encontra-se o Mapa do Texto. Nele é 
possível renomear, inserir, excluir ou alterar a posição do título, parágrafo/seção desde que seja selecionado o conteúdo através do botão estilo "rádio". Na mesma área pode-se abrir a janela de histórico do texto, além de visualizar os participantes do mesmo. Para utilizar as ferramentas de edição do texto, disponíveis logo abaixo da régua de navegação, é necessário que o usuário selecione o "check box" do título, parágrafo/seção que deseja editar e, em seguida, clique na ferramenta desejada. Destacase que cada título, parágrafo/seção contém um link para "Comentários". Este é um recurso para apoiar as discussões quando estiverem presentes diferentes perspectivas acerca da construção de determinado trecho ao longo do texto. Além deste recurso, destacam-se as ferramentas Bate-Papo, Fórum e A2 que foram agregadas ao Editor com a mesma finalidade.

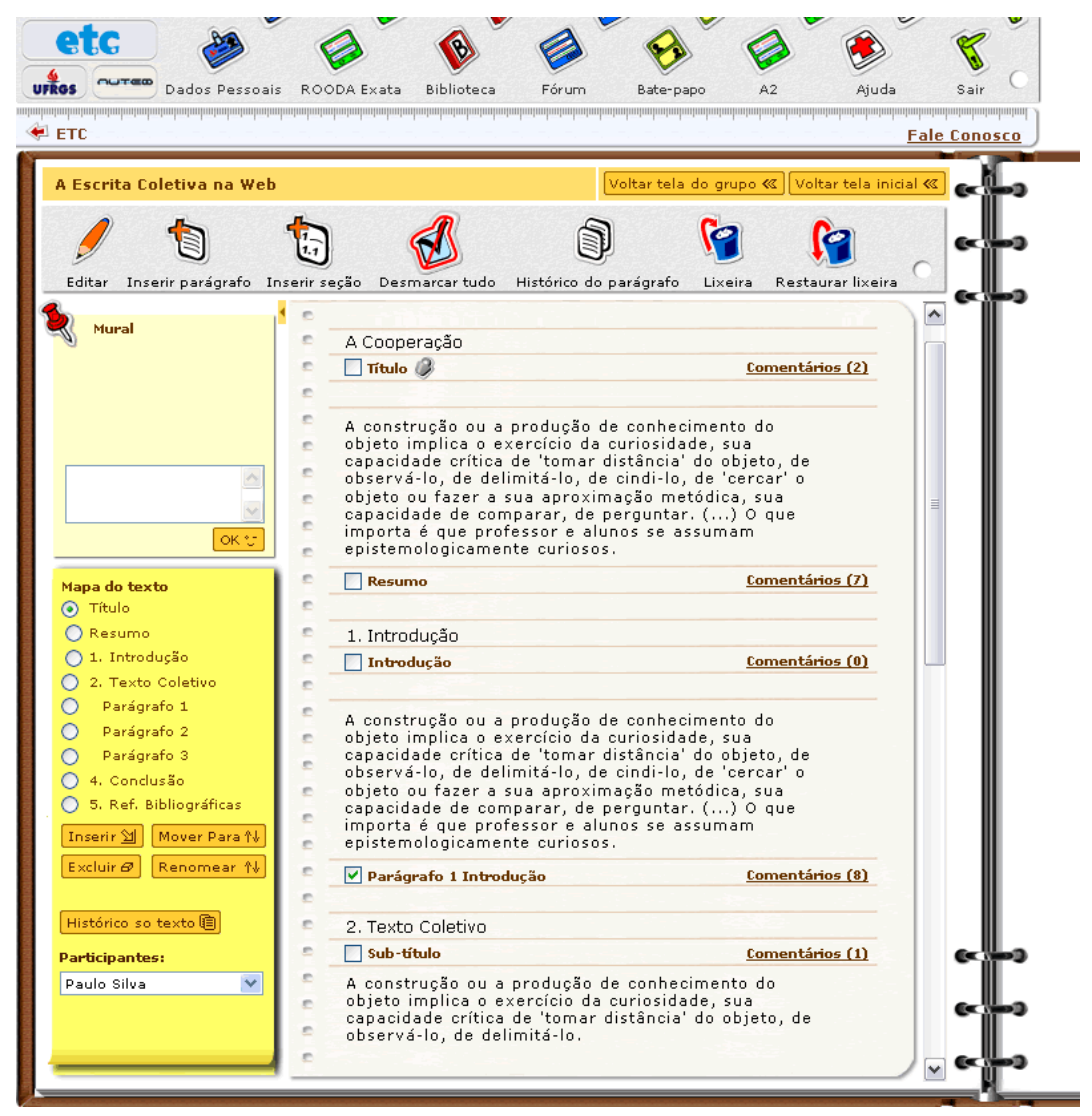

Figura 1 - Tela de edição do texto

A área de edição é ativada logo acima do texto com opções de fontes, tamanho, estilos, inserção de figuras, links (caso tenham sido habilitados na criação do texto) e RoodaExata. A funcionalidade Histórico do Texto destaca-se na medida em que apresenta a participação de cada integrante na construção. Quando selecionado um parágrafo/seção, o Histórico permite visualizar as últimas cinco alterações feitas pelos autores. Cada versão destas alterações pode ser salva localmente.

Com base nesta estrutura de software que o grupo propõe o desenvolvimento do Assistente Virtual para auxiliar a gestão do professor frente a escrita coletiva dos estudantes. 


\section{O Assistente Virtual}

Chamamos de Assistente Virtual aos agentes de interface representados através de personagens que visam aperfeiçoar o processo de interação entre humanos e computadores. Estes personagens são normalmente associados a algum tipo de mecanismo de inteligência artificial que lhes permitem detectar estímulos externos e responder a estes adequadamente. Trata-se do emprego de uma metáfora em que um assistente pessoal colabora com o usuário no mesmo ambiente de trabalho (Maes, 1994). Muitas pesquisas têm investigado o impacto dos assistentes virtuais no desenvolvimento da aprendizagem interativa ${ }^{1}$. Shaw e Johnson (1999) descrevem experimentos com um professor virtual que orienta alunos em atividades interativas online. Craig et al. (2002) investigam diferentes efeitos no processo de aprendizagem de alunos submetidos a personagens estáticos e animados. Sims (2000) apresenta resultados sobre o uso de personagens virtuais no ensino da língua de sinais para crianças com deficiência auditiva. Outros estudos também mostram que a presença da figura humana tem um efeito positivo em experiências interativas com estudantes. Andre et al. (1999) identificaram que estudantes consideram o objeto de estudo menos difícil quando existe a presença de um assistente virtual. $\mathrm{O}$ mesmo estudo mostrou que os estudantes prestam mais atenção aos importantes detalhes da página, em função da presença do assistente.

Aqui, nosso interesse foi de desenvolver um assistente virtual voltado ao apoio do trabalho do professor, e não diretamente orientado à aprendizagem do aluno. Nossa principal motivação foi verificar como os professores têm dificuldade de acompanhar os alunos e promover a interação entre estes em situações de aprendizagem a distância. A perspectiva é de testar este recurso com professores, principalmente de ensino superior, interessados em empregar um editor de texto coletivo para apoiar a produção de seus alunos. Apesar de, tanto o editor quanto o assistente, terem sido pensados para uso a distância, a utilização destes não se restringem a esta modalidade. Sendo assim, a coleta de dados prevê a captura de informações tanto na modalidade a distância, quanto para apoio ao ensino semi-presencial. Num primeiro momento, não configura-se a coleta de dados no ensino presencial pois têm-se especial interesse em analisar a avaliação destes no acesso a distância, já que esta é a modalidade que apresenta a necessidade detectada.

\subsection{Ações Assistente Virtual no ETC}

Em nossa pesquisa foi planejado um modelo de assistente que busca auxiliar o professor em suas tarefas dentro do Editor de Texto Coletivo ETC. As ações deste assistente visam fornecer ao docente suporte para a gestão de sua prática pedagógica. A intenção é agilizar e objetivar o processo de acompanhamento personalizado do estudante, tendo como meta proporcionar que este atinja seu melhor nível de interação no processo de escrita coletiva, bem como de aprendizagem.

As principais características previstas para este assistente virtual são:

$=>$ Através do recurso de text mining, o assistente é capaz de cruzar as contribuições de cada estudante, extraindo conceitos e, a partir destes, construir um grafo que informa ao professor as relações entre conceitos e enfatiza o tipo de contribuição de cada estudante.

\footnotetext{
${ }^{1}$ Quando utilizados em aplicações na educação, tais assistentes são conhecidos como agentes pedagógicos. Optamos por não utilizar esta terminologia na medida em que a principal função do nosso personagem não é voltada à aprendizagem dos alunos mas sim ao apoio às tarefas do professor. Também não utilizamos o termo agente de interface porque tal nomenclatura dá a entender que a modelagem do componente de software desenvolvido segue uma abordagem multiagentes, o que não é o caso.
} 
Esta ação tem como principal objetivo, evidenciar a coerência e apropriação da escrita por cada estudante em relação ao contexto global.

A função mineração de textos para extração de grafos opera sobre textos que devem estar em formato TXT. Para arquivos em outros formatos (PDF, DOC ou outro), através de um pré-processamento é possível convertê-los ao formato TXT.

Partindo-se do arquivo texto, todas as palavras deste são extraídas de forma individual, formando-se uma lista de termos (Gelfand, et al., 1998). A partir desta lista é identificado o tamanho do texto.

$\mathrm{Na}$ seqüência do processo, a lista de palavras é percorrida tentando-se criar palavras combinadas e adicioná-las à lista. Um exemplo disso seria um texto abordando a "Mineração de Dados". Caso esse passo não fosse executado teríamos uma ocorrência elevada dos termos "Mineração" e "Dados", pois o termo "de" seria eliminado por se tratar de um elemento que não expressa significado. Dessa forma saberíamos que o texto trata de "mineração" (talvez relacionada a minérios), e de "dados". Porém, ao localizarmos o termo "Mineração de Dados" e contabilizarmos sua ocorrência, conseguimos identificar um conceito que pode ser importante para a compreensão do conteúdo tratado no texto.

O próximo processo realizado é a eliminação das palavras que não agregam significado, ou não definem qual o assunto que está sendo tratado no texto. Essas palavras são artigos, preposições e outras de baixa ocorrência (Gelfand, et al., 1998); (Brüninghaus, et al., 1998). Em seguida, é realizada a montagem do grafo que representa o texto. Os nodos desse grafo são constituídos pelas palavras que ilustram o assunto desenvolvido (Schenker, 2003). Já os arcos são construídos a partir das relações entre os termos, ou seja, representam as situações nas quais os termos se unem para expressar uma idéia maior (Schenker, 2003a). A seguir, apresentamos um exemplo de texto processado pela ferramenta e transformado no grafo da figura 2.

"Mineração de texto ou Text Mining, também chamado de mineração de dados de texto, refere-se ao processo de obtenção de informação de qualidade a partir de texto em línguas naturais." Após executados os primeiros passos do processamento obteríamos a lista de palavras apresentada na seqüência. Mineração de texto, Text Mining, mineração de dados de texto, processo, obtenção, informação, qualidade, partir, texto, líguas naturais. Essa lista de palavras extraídas é o ponto de partida para a criação do um grafo que representa o texto (Schenker, 2003). O grafo da figura 2 foi construído relacionando-se um termo com os dois termos que aparecem na sua seqüência." 


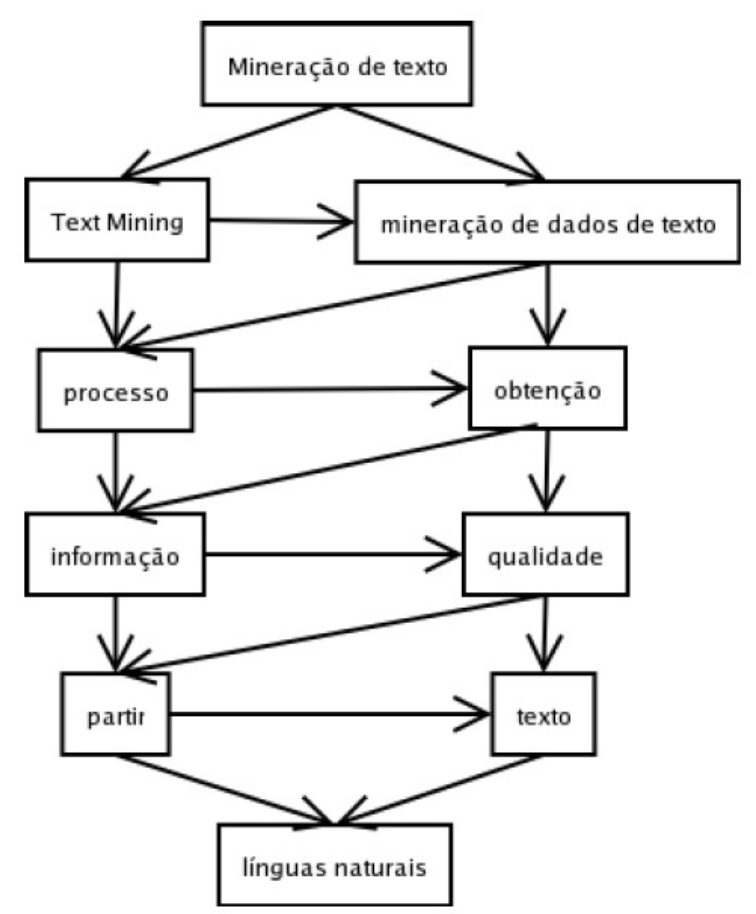

Figura 2 - Grafo extraído da lista de palavras

A representação dos textos dos alunos através de grafos concisos dá suporte ao trabalho de análise e avaliação das contribuições dos alunos pelo professor.

Além disso, o assistente virtual também apresenta um sumário quantitativo informando sobre a contribuição de cada estudante, tal sumário prevê:

$=>$ Número de acessos de cada estudante ao ETC;

=> Alerta informando que o estudante tem acessado o Editor de Texto, mas que não está interagindo com outros estudantes;

$=>$ Alerta informando que o estudante navega pela ferramenta de edição de texto, mas não contribui - (seu objetivo pode ser o de assistir ao processo de construção);

=> Monitora o acesso do usuário aos recursos do Editor no que tange a edição e comunicação com demais participantes. A partir destes dados, o assistente classifica a interação do estudante como sendo não satisfatória, satisfatória ou muito satisfatória a partir de uma escala pré-definida.

Diante disso, entende-se que a compilação das informações descritas acima e apresentadas ao professor, visa fornecer subsídios para sua prática pedagógica diante do processo de aprendizagem e interação de cada aluno.

Para Piaget (1973), o pensamento origina-se da ação e temos na sociedade, um sistema de atividades onde as ações modificam-se umas às outras alcançando formas de equilíbrio. Tais ações são morais, de colaboração, de coação, de comunicação, enfim, uma construção coletiva e de correspondência das operações. A partir da análise dessas interações, deriva a explicação para as representações coletivas, ou interações que modificam a consciência dos indivíduos (Macedo, 2005). Com base nesta perspectiva entende-se que o conjunto das ações propostas para o assistente virtual em questão reflete diretamente no processo de aprendizagem do aluno, uma vez que tal assistente subsidia o professor diante do volume de dados e produções geradas pelos alunos, oferecendo condições para melhor qualificar o desenvolvimento de ações coletivas. 


\section{Considerações Finais}

O trabalho coletivo a distância vem ganhando destaque como um importante recurso para apoiar a aprendizagem. Dentre suas vantagens, destaca-se a flexibilidade geográfica e temporal, as quais se adaptam às necessidades de cada sujeito. A opção por assistentes virtuais no apoio ao trabalho do professor no processo de escrita coletiva deu-se por detectar a necessidade de incorporar princípios e intervenções pedagógicas a este recurso tecnológico.

As funções desempenhadas pelo assistente concebido neste projeto poderiam ser apresentadas ao professor sem a intervenção de um personagem, por exemplo, através de mensagens textuais. No entanto, nossa hipótese é a de que o emprego de um assistente virtual poderia difundir e expandir o uso destas funções por parte dos professores.

Já há alguns anos, cientistas apontam para evidências mostrando que a presença de um personagem em uma interface pode aumentar a confiança do usuário (Rickenberg et al. 2000) e melhorar a comunicação entre homem e máquina através da introdução de estímulos sociais (De Angeli et al. 2001). No entanto, nosso objetivo é disponibilizar as mesmas funções realizadas pelo personagem em uma interface textual, na qual o professor não precise interagir com o assistente virtual.

Quanto ao ETC, entende-se que o software apresenta importantes características e condições de suportar o trabalho coletivo. Nesse sentido, o assistente virtual aqui proposto visa preparar e oferecer melhores subsídios e flexibilidade para uma prática apoiada numa perspectiva de trabalho que enxerga a tecnologia como propulsora de novas possibilidades de cooperação e aprendizagem na prática não presencial.

Este estudo introduziu o desenvolvimento de um assistente virtual para apoiar a escrita coletiva tendo como foco o processo cooperativo e aprendizagem dos sujeitos. Para tanto, o estudo define o tipo de assistente virtual a ser utilizado bem como as ações desempenhadas no Editor de Texto Coletivo. Tais ações apontam no sentido de favorecer a gestão das informações por parte do professor, a fim de auxiliar o mesmo na articulação de suas ações pedagógicas tendo como foco a interação e aprendizagem entre os alunos.

\section{Referências Bibliográficas}

ANDRE, E., RIST, T. and MULLER, J. Employing AI methods to control the behavior of animated interface agents. Applied Artificial Intelligence, Vol. 13, Num. 4-5, May 1999, p. 415-448.

BRÜNINGHAUS Stefanie; D. ASHLEY Kevin. How Machine Learning can be beneficial for Textual CBR. Workshop on Learning for Text Categorization, p.71-74, 1998.

BLOM, J. \& MONK, A. One-to-one e-commerce. who's the one? Proceedings of CHI 2001: Conference on Human Factors in Computing Systems. Seattle, Washington, 31 March - 5 April 2001

BORDINI, R. H.; VIEIRA, R.; MOREIRA, A. F. Fundamentos de sistemas multiagentes. In: SBC2001 - XX Jornada de Atualização em Informática (JAI), Cap. 1 Fortaleza: SBC, 2001. p. 3-41.

CRAIG, S., GHOLSON, B. and DRISCOLL, D. Animated Pedagogical Agents in Multimedia Educational Environments. Journal of Educational Psychology, 2002, Vol. 94, No. 2, 428-434. 
DE ANGELI, A., LYNCH, P. and JOHNSON, G. (2001). "Personifying the e-market: A framework for social agents". Interact 2001 - Eighth IFIP TC.13 Conference on Human-Computer Interaction, Tokyo, Japan, July 9-13, 2001.

ELLIOTT, C.D., RICKEL, J. and LESTER, J. Lifelike Pedagogical Agents and Affective Computing: An Exploratory Synthesis. In M. Wooldridge and M. Veloso (eds.), Artificial Intelligence Today, num. 1600. Lecture Notes in Computer Science, p. 195--212. Springer, 1999.

GELFAND Boris; WULFEKUHLER Marilyn; III Willian F. Punch. Automated Concept Extraction From Plain Text, 1998.

HaLL, P. e WOOD, P. Intelligent Tutoring Systems: A Review for Beginners. Canadian Journal of Educational Communication, 19 (2), pp. 107-123, 1990.

KAASINEN, E. Usability Issues in Agent Applications:What Should the Designer be Aware of. USINACTS 01.06.98, VTT Information Technology, Finland, 1998

MACEDO, A. L. (2005) Aprendizagem em ambientes virtuais: o olhar do aluno sobre o próprio aprender. Porto Alegre: Universidade Federal do Rio Grande do Sul, 2005. 174f. Dissertação (Mestrado em Educação), Programa de Pós-Graduação em Educação, Faculdade de Educação, Universidade Federal do Rio Grande do Sul.

MAES, P. (1994). Agents that reduce work and information overload. Communications of the ACM, 37(7):31-40.

McARTHUR, D., LEWIS, M. e BISHAY, M. The roles of artificial intelligence in education: current progress and future prospects. RAND, Santa Monica, CA, USA, November, 1993.

PIAGET, J. Estudos Sociológicos. Rio de Janeiro: Forense, 1973.

PIAGET, J. Abstração Reflexionante: relações lógico-matemáticas e ordem nas relações espaciais. Porto Alegre: Artes Médicas, 1995.

PICARD, R. Towards Agents that Recognize Emotion. Actes Proceedings IMAGINA, 1998.

REATEGUI, E., BOFF, E., CAMPBELL, J. A. Endowing a Virtual Character with Personalization Capabilities. In: IEEE International Conference on Tools with Artificial Intelligence (ICTAI 06), 2006, Washington, USA, IEEE Press, Novembro 2006.

RICKENBERG, R. e REEVES, B. (2000). The Effects of Animated Characters on Anxiety, Task Performance, and Evaluations of User Interfaces. In Proceedings of $\mathrm{CHI}$ 2000 - Human Factors in Computing Systems. The Hague, Amsterdam, The Netherlands. 1-6 APRIL 2000.

RUSSEL, S.; NORVING, P. Inteligência Artificial. Rio de Janeiro: Elsevier, 2004.

SHAW, E. and JOHNSON, W. L. Pedagogical agents on the Web. In: Proceedings of the the Third International Conference on Autonomous Agents, ACM Press, NY, 1999, 283-290.

SCHENKER, A. Graph-Theoretic Techniques for Web Content Mining. 2003. Tese (Doutorado em Ciência da Computação) - University of South Florida

SCHENKER, A., LAST, M., BUNKE, H., KANDEL, A. Classification of web documents using a graph model. In: ICDAR '03: Proceedings of the Seventh 
International Conference on Document Analysis and Recognition, 2003a, Washington, DC, USA. Anais. IEEE Computer Society, 2003. p.240.

SIMS, E. Virtual Communicator Characters. Computer Graphics. Volume 34, Number 2, May, 2000

WALLACE, David J. She's Only Code and Pixels, but She Can Help You Shop. The New York Times, 20 de setembro de 2000. Disponível em: http://www.nytimes.com/library/tech/00/09/biztech/technology/20wallace.html 